\title{
PTGS2 Over-Expression: A Colorectal Carcinoma Initiator not an Invasive Factor
}

\author{
Tahereh Zahedi ${ }^{1}$, Abasalt Hosseinzadeh Colagar*2, Habibollah Mahmoodzadeh ${ }^{3}$
}

\begin{abstract}
Background: Cyclooxygenase-2 (COX-2) main product is Prostaglandin E2 (PGE2) which cause mitogenesis and inflammation. COX-2 is the product of prostaglandin-endoperoxide synthase 2 (PTGS2) gene expression. COX-2 dysregulation can cause angiogenesis, differentiation, and promotion of cancer and its suppression related to control of the tumor's size, number, and cell shape. This study focused on the association of COX-2 expression with colorectal carcinoma (CRC) among Iranian patients on mRNA level and in the Cancer Genome Atlas Program (TCGA) colon and rectum RNAseq dataset, and its relation with pathological features.

Methods: PTGS2 expression was assayed by quantitative-PCR method from 90 tissue samples collected from 45 participants. The control samples come from the non-tumor area of the same patients. The data analyzed based on $\triangle \Delta \mathrm{Cq}$. The PTGS2-RNAseq data extracted and analyzed by UCSC Xena browser, and its association assessed the occurrence of CRC and invasive-features.

Results: PTGS2 showed very significant over-expression in tumor tissues $(\mathrm{p}<0.0001)$ with an $\mathrm{N}$-fold expression of 2.25. But, there was not any significant association between PTGS2 and CRC invasivepathological features such as Lymphatic, vascular and perineural invasion, the Grades of cancer, and Pathologic-M in both parts of this study.

Conclusions: The increase in PTGS2 is related to the occurrence of CRC among patient samples. But in both part of this study, PTGS2 is not an invasive factor, and it does not affect the cell differentiation of tumors and metastasis. Based on the high $\mathrm{N}$-fold for patient samples, it can be a strong candidate as a CRC initiator biomarker.
\end{abstract}

Keywords: Cyclooxygenase-2, Gene expression profiling, Neoplasm invasion, Prostaglandins, TCGA-data.

\section{Introduction}

Currently, colorectal carcinoma (CRC) is the third universal cause of death by cancer and in general, is one of the most rampant cancers in most of the countries in which Iran is included $(1,2)$. The occurrence of CRC in Iranian men and women is 8.1 to 8.3 and 6.5 to 7.5 per 100,000 , respectively (3). Also, the five years' survival rate of it is $41 \%$ among the Iranian (4).

Since CRC is heterogeneous based on the treatment response and molecular features, the molecular-based studies to characterize the molecular features in malignancy, finding biomarkers, and molecular classification of CRC have improved the individual therapy and reduced the metastatic phase (4-6). Lack of biomarkers, especially in the early stages of CRC is one of the main limitations of preventing it (7). On the other hand, the effect of long term intake of non-steroidal anti-inflammatory drugs (NSAID) on the reduction of CRC promotion risk, especially in familial groups, brought the idea of possible potential of $\mathrm{COX}-2$ as a biomarker for colorectal cancer (8). Also, deeper literature in this matter showed the dysregulation

1: Department of Molecular and Cell Biology, Faculty of Basic Sciences, University of Mazandaran, Babolsar, Mazandaran, Iran.

2: Department of Molecular and Cell Biology, Faculty of Basic Sciences, University of Mazandaran, Babolsar, Mazandaran, Iran. 3: Department of Surgical Oncology, Cancer Institute, Imam Khomeini Hospital Complex, Tehran University of Medical Sciences, Tehran, Iran.

*Corresponding author: Abasalt Hosseinzadeh Colagar; Tel: +9811 35242161; E-mail: ahcolagar@umz.ac.ir.

Received: 10 Nov, 2020; Accepted: 14 Nov, 2020 
of COX-2 in half of the adenomatous polyps which would be counted as the CRC precursors (9).

Cyclooxygenase-2 is the key enzyme of producing prostaglandins (PGs) from arachidonic acid for that the gene is called PTGS2. Among the PGs, the PGE2 is the dominant product of COX-2 which can cause the varieties of malignancy including colon cancer by its role in activating mitogenesis and inflammation (10-13). Even though the COX-2 expression is almost undetectable in most of the normal tissues, but its expression is more frequent in tumorigenic areas $(14,15)$. When the $A P C^{\mathrm{min} /+}$ mouse model was treated by PGE2 the tumor loads had been increased dramatically in both large and small intestines (16). Currently, the amount of urine PGs considered as a biomarker of cancer (11). COX-2 can induce cancer duo to several ways including induces of cancer stem cells like activity, apoptotic resistance, angiogenesis, and metastasis which is approved as the mechanistic aspect of how COX-2 is working (15).

Although several types of researches have shown the effect of PGE2 and overexpression of COX-2 in different types of cancer cells, especially CRC (17-22) but still some studies have not observed the correlation, this controversy is even stronger about its relation with invasion and migration (23-25). So, the aspects of COX-2 in cancer and its relation with CRC that were observed by different studies make it a good point and a potent biomarker but it needs more studies on the relation of the COX2 expression in different populations.

In the present study, because of the lack of enough studies on COX-2 expression in Iranian cancer patients, especially in CRC, despite of its potent as a prognostic biomarker candidate, the purpose was assessing of correlation of PTGS2 expression with colorectal cancer in patients' tissues, and the CRC pathological features. Furthermore, we evaluated the PTGS2 expression of colon and rectum samples from TCGA and its correlation with some of the invasive pathological features by use of TCGACOAD and READ data-sets.

\section{Materials and methods}

The participants were the colorectal cancerous patients (2012 to 2016) whose been attended for the tumor removal surgery in Imam Khomeini hospital (Tehran, Iran). Forty-five patients have participated in this study and all of them have been adults (ID Number of Ethical Committee: \#IR.UMZ.REC.1397.091). The tumor area and the non-tumor adjacent tissues have been used as case and control groups. The tissues have been frozen immediately after surgery in a liquid nitrogen tank. The prevalence of colorectal cancer in Iranian is 8.3 in 100,000 . So based on the standard formula the number of samples in our study is proper for this study.

The patients have been classified based on the Bloom-Richardson system which is calculating the grade by adding up the scores of nuclear pleomorphism and mitotic count, and TNM staging system. The cell nuclei uniformity and the number of divided cells in the 10x high power microscope filed would be assessed in this method (26). Also, the situation of each patient was checked based on the invasion of tumor tissues to other tissues like Lymphatic, vascular, perineural, and perineal.

\section{RNA isolation and RT $q-P C R$}

Total RNA was extracted by the standard TRIzol $^{\mathrm{TM}}$ Plus kit protocol. In order to check the quantity and quality of isolated RNA, the OD reading by NanoDrop and gel electrophoresis have been used. For cDNA the standard protocol of Thermo Scientific ${ }^{\mathrm{TM}}$ RevertAid $^{\mathrm{TM}}$ first-strand cDNA kit was applied.

The COX-2 (PTGS2), and ACT $\beta$ were quantified in 3 repeats by CYBR Premix Ex Taq II (the primers of PTGS2 (COX-2), and ACT $\beta$ (Gene Control) has been designed by Primer 3 [http://primer3.ut.ee/]), and Applied Biosystems 7500/7500 Fast Real-Time PCR. To be sure of no contamination, negative controls were applied. The qPCR applied with the blinding procedure. The forward and reverse primers sequences of $\mathrm{COX}-2$ were 5'AGGAGGTCTTTGGTCTGGTG-3' and 5'ACTGCTCATCACCCCATTCA-3' respectively. To analyze the $P T G S 2$ expression 
data, after normalising it to $A C T \beta$ expression, fold expression $\left(2^{-\Delta \Lambda \mathrm{Cq}}\right.$ ) and $\log _{2}$ (Fold expression) were used (27).

\section{PTGS2 expression data extraction and RNA-seq analysis}

To generate the PTGS2 expression data of colon and rectum cancer and normal tissues, we used the UCSC Xena browser (Xena.ucsc.edu). To analyze the association of PTGS2 expression with CRC, the data of TCGA-COAD (331-samples) and READ (103-samples) were compared with TCGA-solid normal tissues, and the dataset of RNA-Seq by Expectation Maximization (RSEM) expected-count normalized by DESeq2 normalization. The correlation of PTGS2 expression with pathological features such as Pathological-M and lymphatic invasion among 2236 TCGA-COAD-READ samples analyzed. The PTGS2 level expressed based on $\log _{2}$ (normcount+1) unit (28).

\section{Statistical analysis}

After normal distribution of $\log _{2}$ (Fold Expression) in general has been checked, the Mann-Whitney test was used for statistical analysis of COX-2 expression between case and control groups. For the association of COX-2 expression with the other factors like stages and invasions, depend on the character the proper nonparametrical analysis (Mann-Whitney and Kruskal-Wallis, and Dunn's multiple comparison test) has been used and $* \mathrm{p} \leq 0.05, * * \mathrm{p} \leq 0.01$, $* * * p \leq 0.001, * * * * p \leq 0.0001$ were considered as significant differences. Results are presented as median \pm SEM by Prism 8 .

\section{Results \\ General characters and quantitative analysis}

All of the general characters of the samples are presented in Table 1. The COX-2 showed a highly significant overexpression (N-Fold Expression $=2.25$ ) in tumor samples compare to normal tissues (Mann-Whitney $U=354, \mathrm{p}<$ 0.0001 two-tailed). This significant overexpression observed in all of the regions based on the primary site, except the Cecum groups with non-significant overexpression (Fig. 1). In both of non-tumor group and tumor tissues, there were not any significant COX-2 expression differences within the primary site of the samples (Tumor $p=0.256$; Normal $p=$ 0.427). In both groups, the most underexpressions belonged to the cecum.

Table 1: Groups characteristics analysis.

\begin{tabular}{lll}
\hline & Characters & \multicolumn{1}{c}{ No (Percent \%) } \\
\hline \multirow{3}{*}{ TNM stages } & Stage 0 & $3(6.7 \%)$ \\
& Stage I & $7(15.6 \%)$ \\
& Stage II & $9(20.0 \%)$ \\
& Stage III & $11(24.4 \%)$ \\
& Stage IV & $3(6.7 \%)$ \\
Mloom Richardson & Grade I & $12(26.6 \%)$ \\
\hline \multirow{3}{*}{ Donor's statue } & Grade II & $14(31.1 \%)$ \\
& Grade III & $17(37.8 \%)$ \\
& Grade IV & $2(4.5 \%)$ \\
& Alive & $11(24.5 \%)$ \\
\hline \multirow{3}{*}{ Histology } & Deceased & $35(77.8 \%)$ \\
& Adenocarcinoma & $10(22.2 \%)$ \\
\hline & Adenosquamous carcinoma & $30(66.7 \%)$ \\
& Medullary carcinoma & $10(22.2 \%)$ \\
& Mucinous carcinoid & $1(2.2 \%)$ \\
& Mucinous (colloid) & $3(6.7 \%)$ \\
\hline & adenocarcinoma & \\
\hline
\end{tabular}



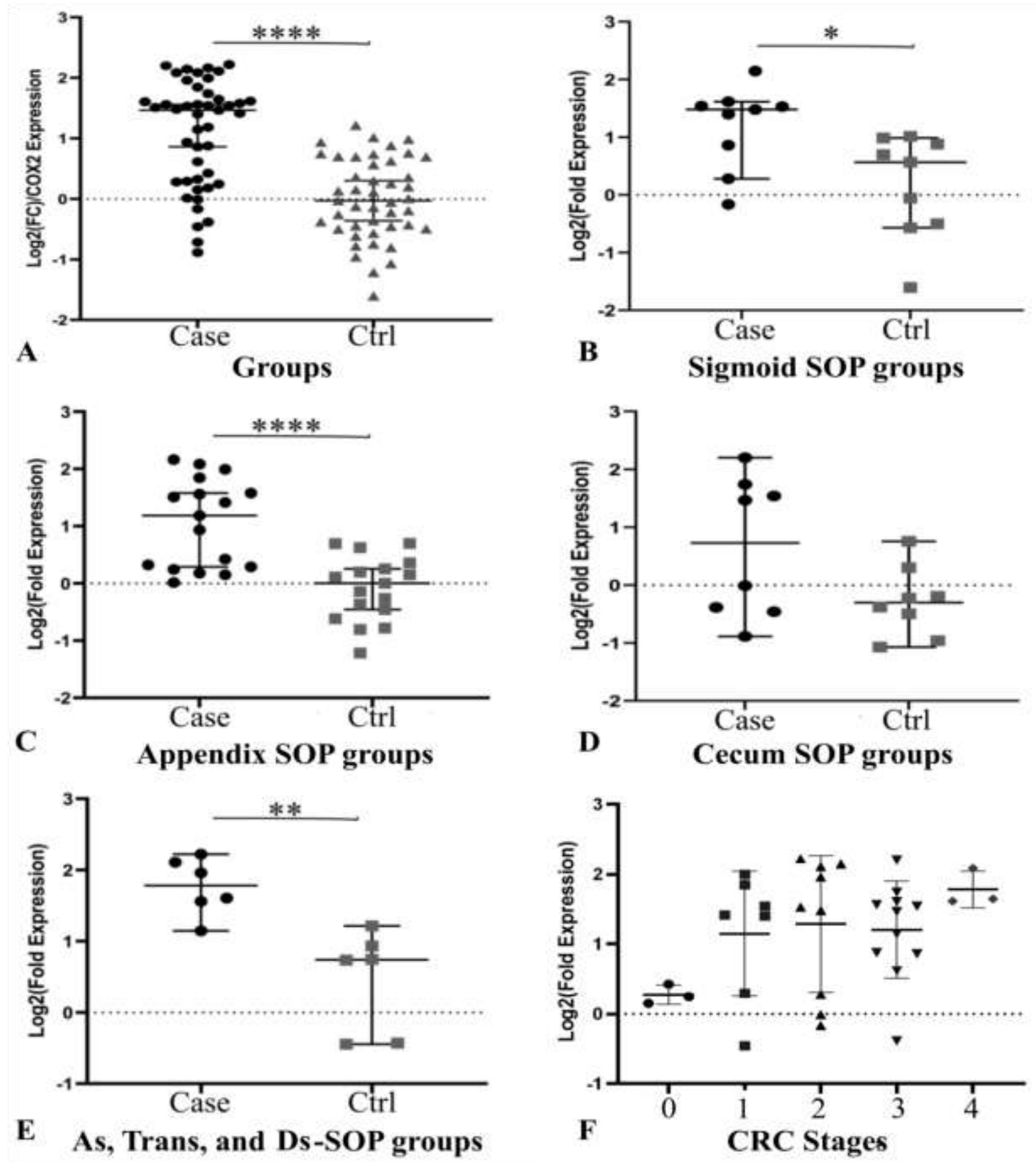

Fig. 1. Comparison of COX-2 expression in patient and control groups, and cases stages of cancer. A) The Cox-2 is significantly overexpressed in the tumor samples in general $(\mathrm{p}<0.0001)$ in individual chart. B) The sigmoid primary site's COX-2 expression is significantly higher $(\mathrm{p}=0.0188)$. C) There is highly significant COX-2 overexpression in Appendix tumor group compare to Appendix control ( $p<0.0001)$. D) The cecum primary site's $p$-value is 0.1605 . E) The p-value of the samples that were located from ascending, transverse and descending (As, Trans, and Ds) colon is 0.0043 that represent highly significant differences. F) Increase of COX-2 expression due to increase of stages is observable but it is not statistically significant.

As it is displayed in Figure 1-F, even though the level of COX-2 expression is going up from lower stages to the higher stages of CRC, yet it was not significant $($ Exact $\mathrm{p}=0.19, \mathrm{df}=4, \mathrm{No}=$ 33). Also, the COX-2 expression has no participation in cell differentiation either (Fig. 2). 


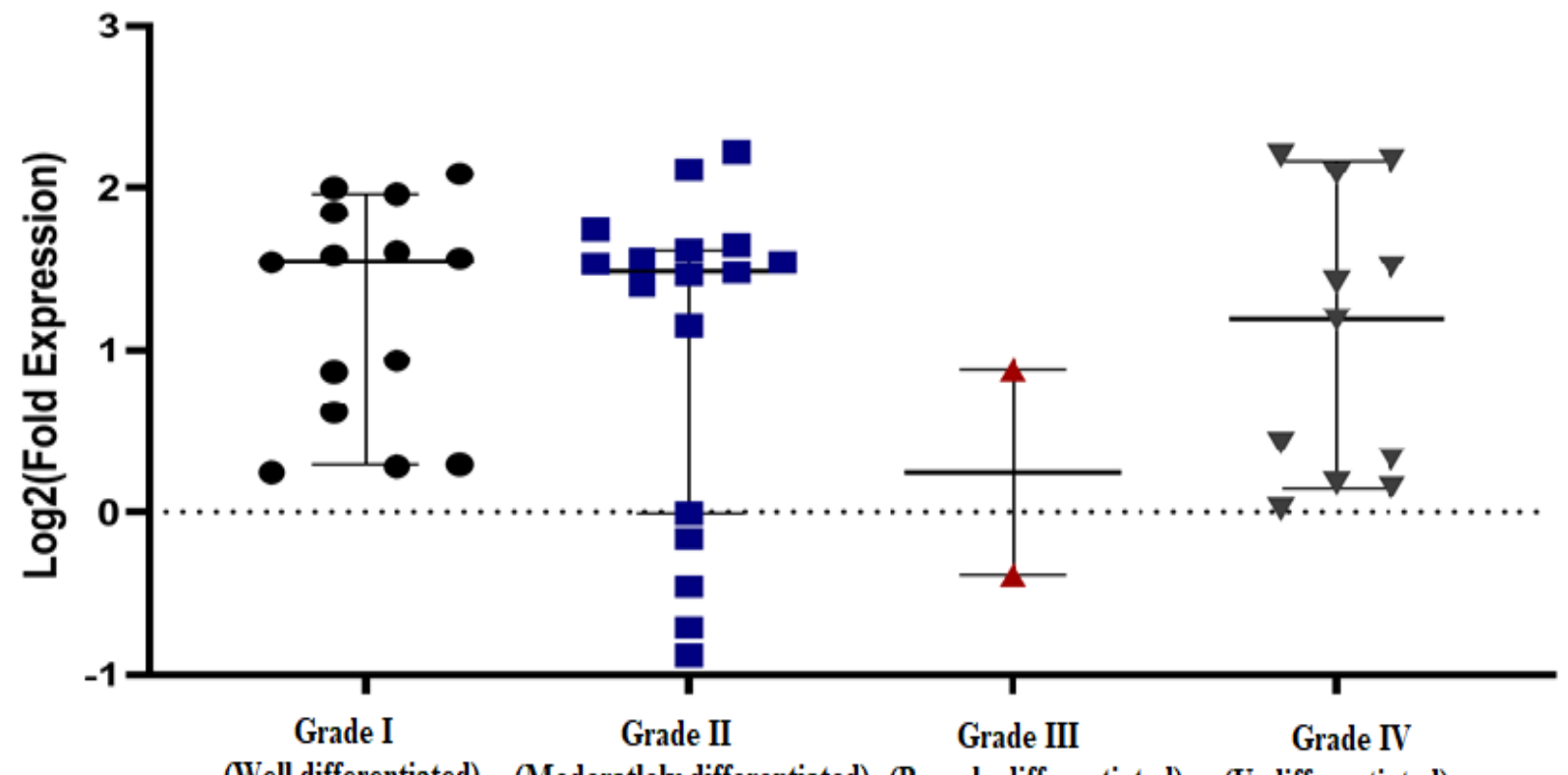

(Well differentiated) (Moderatlely differentiated) (Poorely differentiated) (Undifferentiated)

\section{CRC Grades}

Fig. 2. The COX-2 expression in different Grades of CRC; The COX-2 expression is not significantly difference between different grades of cell differentiation.

The association of COX-2 with some other malignancy factors including lymphatic invasion, vascular invasion, and the statue of patients examined, even though there was a real significant difference between the tumor and nontumor samples but there weren't any between the lymph-vascular invasion and COX-2 expression; besides the lymphatic extension positive represents lower expression compared to the noninvasive. However, COX-2 almost overexpressed in the invasive perineural and deceased groups related to the non-invasive and alive (Table 2).

Table 2. The COX-2 mRNA expression and its association with different malignancy characterize.

\begin{tabular}{|c|c|c|c|c|c|}
\hline \multicolumn{2}{|c|}{ Name of groups } & No & Mean \pm SD & Median & p-value \\
\hline \multirow[t]{2}{*}{ General } & Case & 45 & $1.088 \pm 0.878$ & 1.466 & $<0.0001 *$ \\
\hline & Control & 45 & $-1.1 \mathrm{e}-10 \pm 0.67$ & -0.029 & \\
\hline \multirow[t]{5}{*}{ Stages } & Stage 0 & 3 & $0.274 \pm 0.1397$ & 0.2461 & 0.192 \\
\hline & Stage I & 7 & $1.148 \pm 0.8957$ & 1.416 & \\
\hline & Stage II & 9 & $1.284 \pm 0.9771$ & 1.531 & \\
\hline & Stage III & 11 & $1.203 \pm 0.6964$ & 1.466 & \\
\hline & Stage IV & 3 & $1.783 \pm 0.2631$ & 1.646 & \\
\hline \multirow[t]{2}{*}{ Grades } & Low Grades & 31 & $1.118 \pm 0.876$ & 1.531 & 0.464 \\
\hline & High Grades & 13 & $0.9353 \pm 0.888$ & 0.876 & \\
\hline \multirow[t]{2}{*}{ Vascular invasion } & Positive & 23 & $1.185 \pm 0.798$ & 1.561 & 0.844 \\
\hline & Negative & 17 & $1.215 \pm 0.781$ & 1.416 & \\
\hline \multirow[t]{3}{*}{ Lymphatic invasion } & Positive (NOS) & 21 & $1.365 \pm 0.693$ & 1.581 & 0.117 \\
\hline & Positive (Extensive) & 9 & $0.811 \pm 0.747$ & 0.4261 & \\
\hline & Negative & 8 & $1.242 \pm 0.844$ & 1.434 & \\
\hline \multirow[t]{2}{*}{ Perineural invasion } & Positive & 6 & $1.54 \pm 0.98$ & 0.46 & 0.086 \\
\hline & Negative & 34 & $1.043 \pm 0.84$ & 0.27 & \\
\hline \multirow[t]{2}{*}{ Extracapsular node extension } & Positive & 4 & $1.63 \pm 0.736$ & 1.85 & 0.13 \\
\hline & Negative & 35 & $1.079 \pm 0.824$ & 1.40 & \\
\hline \multirow[t]{2}{*}{ Statue of patients } & Alive & 34 & $1.087 \pm 0.84$ & 1.434 & 0.170 \\
\hline & Deceased & 9 & $1.508 \pm 0.499$ & 1.581 & \\
\hline
\end{tabular}


PTGS2 expression Multi-Omics and

\section{bioinformatics analysis}

Based on the analysis of TCGA-COAD and READ versus TCGA-solid normal tissues, the PTGS2 expression of primary tumor samples have not any significant differences with solid normal tissues (Fig. 3). Though among 103 rectum samples the solid normal tissue $\left(\log _{2}\right.$

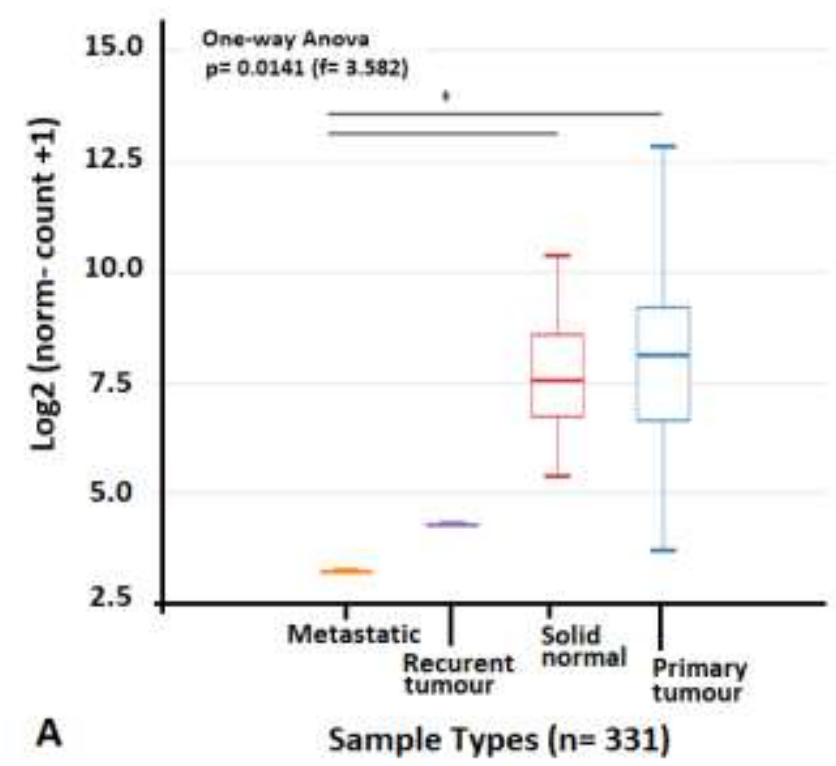

Fig. 3. The PTGS2 expression comparison of TCGA-COAD, -READ and normal solid tissues. A) PTGS2 expression of TCGACOAD-primary tumor and TCGA-Solid Normal Tissues were not any significant differences; the metastatic sample $(n=1)$ showed significant lower expression compare to the other 2 groups. B) PTGS2 expression of TCGA-READ and TCGA-Solid Normal Tissues does not show significant differences.

The correlation of PTGS2 expression with some pathological features evaluated. In here, we specifically focused on metastasis and lymphatic invasion as two invasive factors. There was not any significant correlation between PTGS2 expression and invasive-pathological features (Fig. 4A-B). But READ datasets presented significant differences of PTGS2 expression among samples with the history of colon cancer compared to without it (Fig. 4C), while there is no association between history-of-colon-cancer with PTGS2 level among COAD samples.

\section{Discussion}

The COX-2 is an inducible type of its family and its expression is mostly limited to the central nervous system, and women reproduction system while its overexpression in the tumor environment exhibited in different studies $(14,15,29)$. On the other hand, there is the PGE2 as the main product

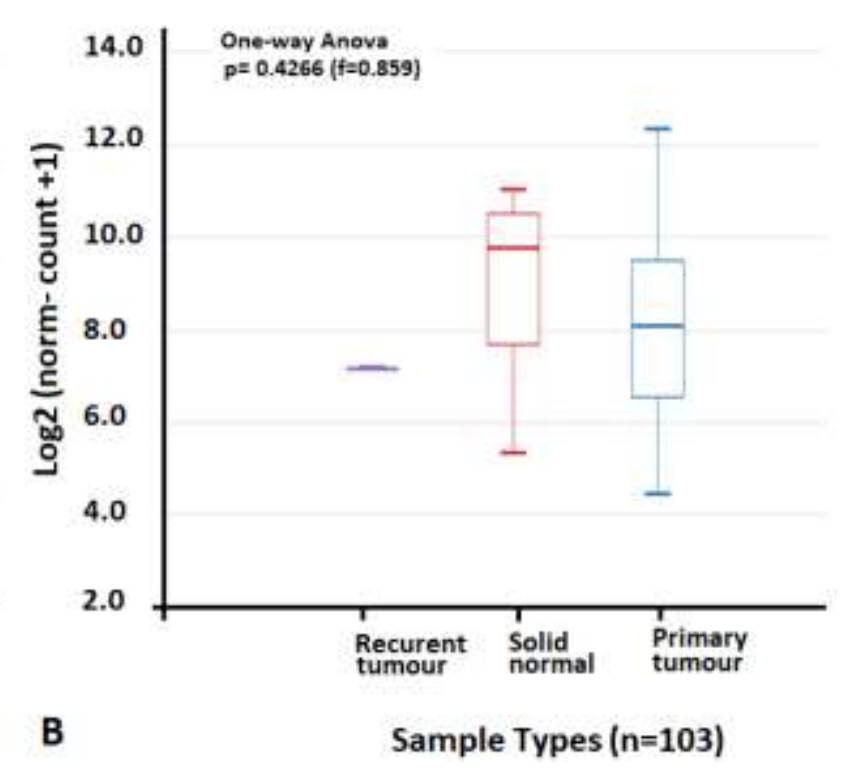

norm-count $+1=9.75$ ) represented higher (nonsignificant) PTGS2 expression compares to the primary tumor $\left(\log _{2}\right.$ norm-count $\left.+1=8.07\right)$, but it is the opposite in 331 colon samples (Solidnormal $\log _{2}$ norm-count $+1=7.54$; Primary-tumor $\log _{2}$ norm-count $+1=8.09$ ).

of $\mathrm{COX}-2$, which is the cause of inflammation and several aspects of malignancies such as cell proliferation, angiogenesis, migration, and invasiveness. Also, inflammation itself can ignite the beginning of the adenocarcinoma and the carcinogenesis process. As we can see in previous studies removing of $\mathrm{COX}-2$ is reducing the number of polyps in $\mathrm{APC}^{\Delta 716}$ epigenetic mice models (30). In some of the studies on patient samples, the relation of $\mathrm{COX}-2$ protein overexpression with tumor recurrence, invasion, and metastatic proved, while some others have not shown these correlations in protein or mRNA $(10,24,31,32)$.

Based on the results of this study on patient samples, we can see the very significant association of COX-2 overexpression with the tumorigenesis by the fold expression of 2.25 . The overexpression of PTGS2 mRNA is obvious in malignant tissues compared to normal tissues 


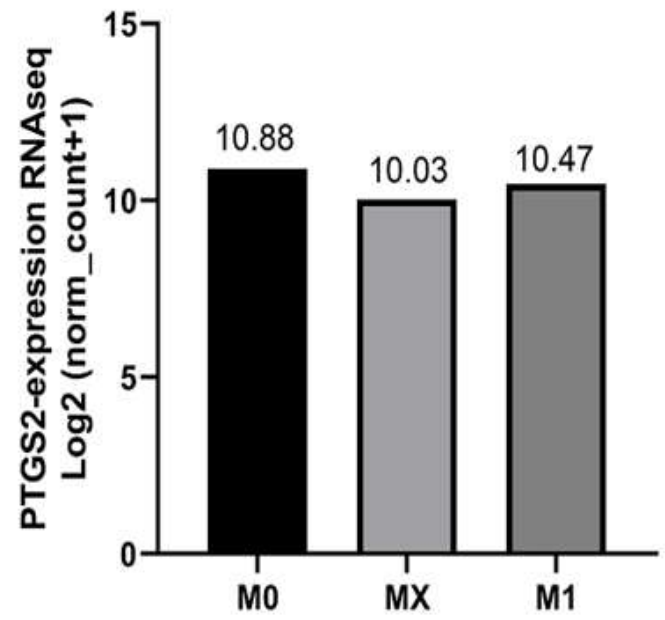

A

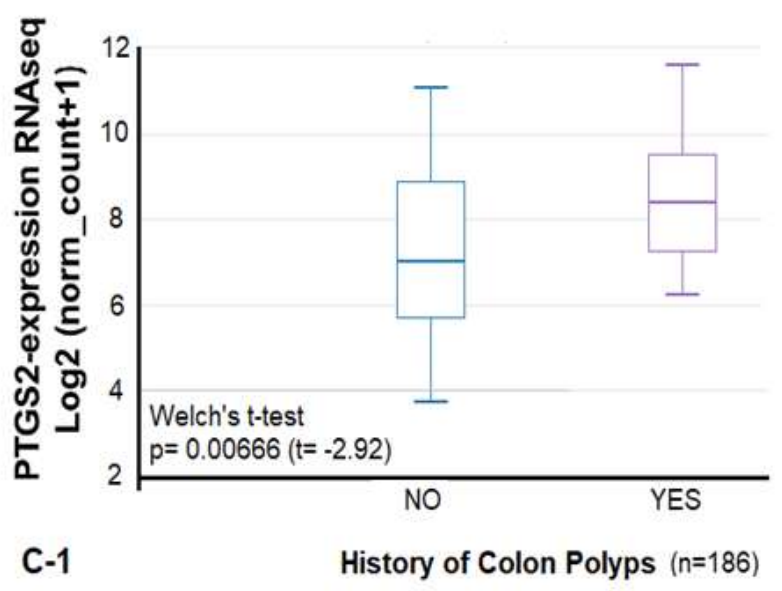

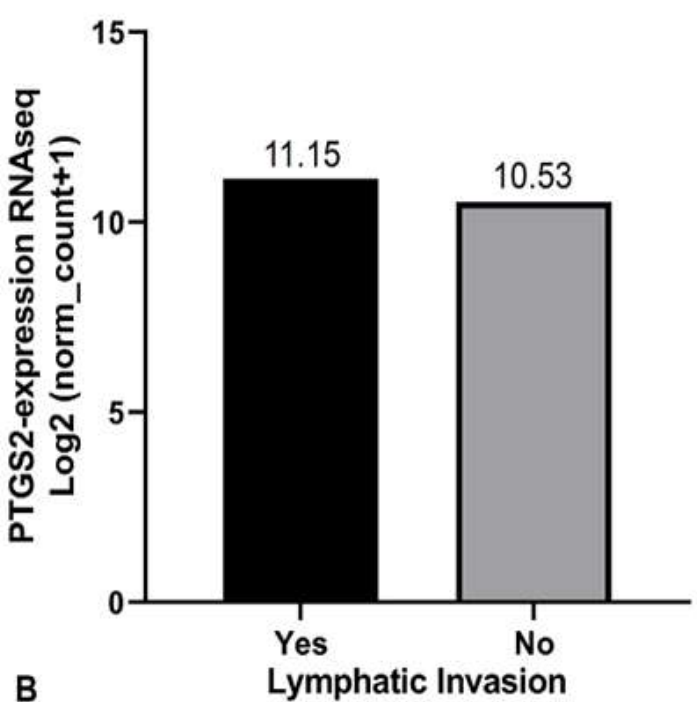

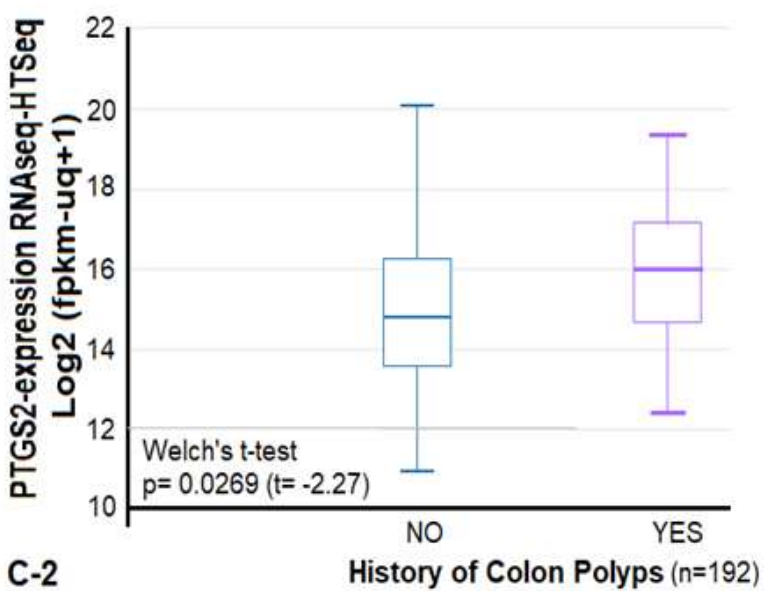

Fig. 4. Correlation of PTGS2 expression with pathological features in TCGA-COAD and -READ. A) Among 1519 samples, the PTGS2 expression did not represent any significant correlation with Pathologic-M feature. B) The PTGS2 expression in lymphatic invasive group is close to the non-invasive one. C) The TCGA-READ datasets showed significant higher PTGS2 expression in group with the history of colon polyps.

which belonged to the same participants, but in between the invasive and non-invasive groups, it is not significant. So, these results are related to starting of tumorigenesis rather than angiogenesis and invasion. On the other hand, the analysis of TCGA colon and rectum datasets did not show significant differences in PTGS2 expression, not as an invasive factor and not as a tumorigenesis one. But among the TCGA-READ samples that have history of colon polyps, the expression of PTGS2 was significantly higher. Therefore, in this study, the results are more met with the inflammatory impact of PTGS2 at the beginning of CRC, and several analysis by the current study make it clear that PTGS2 overexpression has no participation in invasion and metastasis of CRC in patient samples. Also, there is no difference among the non-differentiated group and welldifferentiated ones, and the TCGA data analysis did not show any differences between metastatic groups and M0. When we are looking at the results of PTGS2 expression in the deceased group, the average of PTGS2 expression is higher than the alive group though it is not significant either.

The results of this study on patient samples are following the previous studies on COX-2 mRNA expression in another population and the TCGA data analysis that was done before but our results on TCGA datasets are different from the previous 
study $(1,32,33)$. Though the other study on adenocarcinoma in Iranian patients, the COX-2 protein expression showed increasing as same as our results; but they observed a significant relationship between COX-2 expression and lymph node invasion unlike our study at both level of patient samples and TCGA analysis (31). In the other study, COX-2 expression was assessed based on semi-quantitative method while, our study was quantitative base. In the present study, the assessment was done in mRNA level, but the aforementioned study was on the protein which can be another reason for the contrary on the results. Also, in this study, there are no differences in the COX-2 expression among the 4 Grades. Hence, the loss of cell differentiation that is one of the main factors for further development of the tumor is not related to the COX-2 expression based on the current study.

In general, there are conflicting results in the matter of COX-2 association with the clinicopathological factors of the patients in CRC. Also, one of the previous studies on cell lines has shown the higher expression of COX-2 on the differentiated carcinoma group same as us. By an overview of various studies on both cell lines and tissues, and the current study, the COX-2 overexpression affects the initial cell growth (10,31-36). Furthermore, based on several studies

\section{References}

1. Zhoe FF, Huang R, Jiong J, Zong XH, Zou SQ. Correlated non-nuclear COX-2 and low HER2 expression confers a good prognosis in colorectal cancer. Saudi J Gastroenterol. 2018;24(5):301-306.

2. Rafiemanesh H, Pakzad R, Abedi M, Kor Y, Moludi J, Towhidi F, et al. Colorectal cancer in Iran: epidemiology and morphology trends. EXCLI J. 2016;15:738-744.

3. Moghimi-Dehkordi B, Safaee A, Zali M. Prognostic factors in 1,138 Iranian colorectal cancer patients. International Journa Of Colorectal Disease. 2008;23(7):683-688.

4. Dolatkhah R, Sami MH, Jabbarpour-Bonyadi M, Asvadi-Kermani I, Farassati F, Dastgiri S. Colorectal cancer in Iran: molecular epidemiology and screening strategies. J Cancer Epidemiol. 2015:643020. the COX-2 inhibitors have a significant effect on cell growth and proliferation with which the result of this study on patient samples is consistent $(37,38)$. Furthermore, we showed that COX-2 overexpression on mRNA level has no role in the further development of CRC.

In conclusion the results of the current study, are approving the highly significant relation of COX-2 mRNA expression and occurrence of $\mathrm{CRC}$, and there is up-regulation in tumor tissues for COX-2 in Iranian populations. The TCGACOAD and -READ analysis showed no overexpression related to CRC. But the PTGS2 overexpressed in TCGA-READ samples with a history of colon polyps that matched with COX-2 inflammatory effect. Therefore, it is a highly potent candidate as a CRC biomarker at early grades of tumorigenesis among Iranian patients, but the new finding is that it doesn't affect the further development of invasive features of tumors based on both steps of this study.

\section{Acknowledgements}

Authors would like to thank the management of the University of Mazandaran, for supporting and encouragement to carry out this work and Hadiseh Mohammadpour for her technical assistance.

There is no conflict of interest exists.

5. Sveen A, Bruun J, Eide PW, Eilertsen IA, Ramirez L, Murumagi A, et al. Colorectal cancer consensus molecular subtypes translated to preclinical models uncover potentially targetable cancer cell dependencies. Clin Cancer Res. 2018;24(4):794-806.

6. Malekzadeh R, Bishesari F, Mahdavinia M, Ansari R. Epidemiology and molecular genetics of colorectal cancer in Iran: a review. Arch Iran Med. 2009;12(2):161-9.

7. Dixon DA, Blanco FF, Bruno A, Patrignani P. Mechanistic aspects of COX-2 expression in colorectal neoplasia. Recent Results Cancer Res. 2013;191:7-37.

8. Gupta RA, DuBois RN. Colorectal cancer prevention and treatment by inhibition of cyclooxygenase2. Nat Rev Cancer. 2001;1(1):11-21. 
9. Periera C, Quieros S, Galaghar A, Sousa H, Marcos-Pinto R, Pimentel-Nunes P, et al. Influence of genetic polymorphisms in prostaglandin E2 pathway (COX-2/HPGD/SLCO2A1/ABCC4) on the risk for colorectal adenoma development and recurrence after polypectomy. Clin Transl Gastroenterol. 2016;7(9):e191.

10.Batista WR, Santos G, Vital FMR, Matos D. Immunoexpression of TS, p53, COX2, EGFR, MSH6 and MLH1 biomarkers and its correlation with degree of differentiation, tumor staging and prognostic factors in colorectal adenocarcinoma: a retrospective longitudinal study. SaoPaulo Med J. 2019;137(1):33-38.

11.Cui Y, Shu XO, Li HL, Yang G, Wen W, Gao YT, et al. Prospective study of urinary prostaglandin E2 metabolite and pancreatic cancer risk. Int $\mathbf{J}$ Cancer. 2017;141(12):2423-2429.

12.Greenhough A, Smartt HJM, Moore AE, Roberts HR, Williams AC, Paraskeva C, et al. The Cox-2/PGE-2 pathway: key roles in the hallmarks of cancer and adaptation to the tumour microenvironment. Carcinogenesis. 2009;30(3):377-86.

13. Cox DG, Pontes C, Guino E, Navarro M, Osorio A, Canzian F, et al. Polymorphisms in prostaglandin synthase 2/cyclooxygenase 2 (PTGS2/COX2) and risk of colorectal cancer. $\mathrm{Br} \mathrm{J}$ Cancer. 2004;91(2):339-43.

14. Gurram B, Zhang S, Li M, Li H, Xie Y, Cui H, et al. Celecoxib conjugated fluorescent probe for identification and discrimination of cyclooxygenase2 enzyme in cancer cells. Anal Chem. 2018;90(8):5187-5193.

15.Hashemi Goradel N, Najafi M, Salehi E, Farhood B, Mortezaee K. Cyclooxygense-2 in cancer: a review. Journal of Cellular Physiology. 2018;234(5).

16.Wang D, Wang H, Shi Q, Katkuri S, Walhi W, Desvergne B, et al. Prostaglandin E2 promotes colorectal adenoma growth via transactivation of the nuclear peroxisome proliferator-activated receptor delta. Cancer Cell. 2004;6(3):285-95.

17.Gasparini G, Longo R, Sarmiento R, Morabito AN. Inhibitors of cyclooxygenase 2: a new class of anticancer agents?. Lancel Oncol. 2003;4(10):605-15. 18. Soslow RA, Dannenberg AJ, Rush D, Woerner $\mathrm{BM}$, Khan $\mathrm{KN}$, Masferrer $\mathrm{J}$, et al. Cox-2 is expressed in human pulmonary, colonic, and mammary tumors. Cancer. 2000;89(12):2637-45. 19.Raj V, Bhaauria AS, Singh AK, Kumar U, Rai A, Keshari AK, et al. Novel 1,3,4-thiadiazoles inhibit colorectal cancer via blockade of IL-6/COX2 mediated JAK2/STAT3 signals as evidenced through data-based mathematical modeling. Cytokine. 2019;118:144-159.

20.Mortezaee K. Human hepatocellular carcinoma: protection by melatonin. Journal of Cellular Physiology. 2018;233(10):6486-508.

21.Ohtsuka J, Oshima H, Ezawa I, Abe R, Oshima M, Ohki R. Functional loss of P53 cooperates with the in vivo microenvironment to promote malignant progression of gastric cancers. Sci Rep. 2018;8(1):2291.

22.Montezuma MAP, Fonsera FP, Benites BM, Soares CD, do Amaral-Silva GK, de Almeida OP, et al. COX-2 as a determinant of lower disease-free survival for patients affected by ameloblastoma. Pathol Res Pract. 2018;214(6):907-913.

23.Destefanis F, Fiorito V, Altruda F, Tolosano E. Investigating the connection between endogenous Heme accumulation and COX-2 activity in cancer cells. Front Oncol. 2019;9:162.

24. Kim SH, Ahn BK, Paik SS, Lee KH. Cyclooxygenase-2 Expression Is a Predictive Marker for Late Recurrence in Colorectal Cancer. Gastroenterol Res Pract. 2018:7968149.

25.Xu Z, Choudhary S, Voznesensky O, Mehrota M, Woodard M, Hansen M, et al. Overexpression of COX-2 in human osteosarcoma cells decreases proliferation and increases apoptosis. Cancer Res. 2006;66(13):6657-64.

26. Al-Kuraya K, Schraml P, Torhorst J, Tapia C, Zaharieva B, Novotny H, et al. Prognostic relevance of gene amplifications and coamplifications in breast cancer. Cancer Res. 2004;64(23):8534-40.

27.Livak KJ, Schmittgen TD. Analysis of relative gene expression data using real-time quantitative PCR and the 2(-Delta Delta C(T)) Method. Methods. 2001;25(4):402-8.

28. Goldman MJ, Craft B, Hastie M, Repecka K, McDade F, Kamath A, et al. Visualizing and interpreting cancer genomics data via the Xena platform. Nat Biotechnol. 2020;38(6):675-678.

29. Wang D, DuBois RN. Eicosanoids and cancer. Nat Rev Cancer. 2010;10(3):181-93. 
30. Oshima M, Dinchuk JE, Kargman SL, Oshima H, Hancock B, Kwong E, et al. Suppression of intestinal polyposis in APC delta 716 knockout mice by inhibition of cyclooxygenase 2 (COX-2). Cell. 1996;87(5):803-9.

31.Habibollahi P, Jamshidiha M, Daryani NE, Jahanzad I, Ghahremani MH, Ostad SN. Correlation between inducible nitric-oxide synthase and cyclooxygenase- 2 expression in human colorectal adenocarcinoma: a cross-sectional study. Pathol Oncol Res. 2010;16(3):327-35.

32.Fujita T, Matsuni M, Takaku K, Uetake H, Ichikawa W, Taketo MM, et al. Size- and invasiondependent increase in cyclooxygenase 2 levels in human colorectal carcinomas. Cancer Res. 1998;58(21):4823-6.

33.Negi RR, Rana SV, Gupta V, Gupta R, Chadha VD, Prasad KK, et al. Over-Expression of Cyclooxygenase-2 in Colorectal Cancer Patients. Asian Pac J Cancer Prev. 2019;20(6):1675-1681.

34. Tomozawa S, Tsuno NH, Sunami E, Hatano K,
Kitayama J, Osada T, et al. Cyclooxygenase-2 overexpression correlates with tumour recurrence, especially haematogenous metastasis, of colorectal cancer. $\mathrm{Br} \mathrm{J}$ Cancer. 2000;83(3):324-328.

35.Sheng H, Shao J, Kirkland SC, Isakson P, Coffey RJ, Morrow J, et al. Inhibition of human colon cancer cell growth by selective inhibition of cyclooxygenase-2. J Clin Invest. 1997;99(9):2254-9. 36. Masferrer JL, Leahy KM, Koki AT, Zweifel BS, Settle SL, Woerner BM, et al. Antiangiogenic and antitumor activities of cyclooxygenase-2 inhibitors. Cancer Res. 2000;60(5):1306-11.

37. Umezawa S, Higurashi T, Komiya Y, Arimoto J, Horito N, Kaneko T, et al. Chemoprevention of colorectal cancer: past, present, and future. Cancer Sci. 2019;110(10):3018-3026.

38.Zare M, Soltanzadeh H, Narimani R. Plasma level of miRNA-7, miRNA-409 and miRNA-93 as potential biomarkers for colorectal cancer. J Genet Resour. 2019;5(1):9-16. 